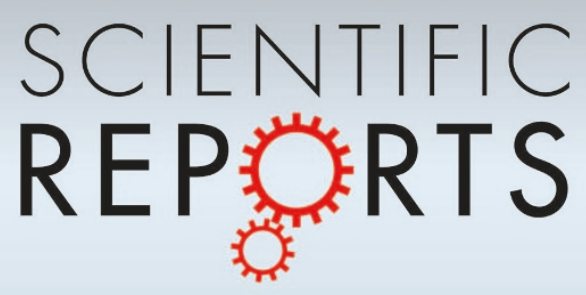

OPEN

SUBJECT AREAS:

EVOLUTIONARY

DEVELOPMENTAL

BIOLOGY

DEVELOPMENTAL BIOLOGY

PSYCHOLOGY

ANIMAL BEHAVIOUR

Received

30 April 2013

Accepted

25 September 2013

Published

19 November 2013

Correspondence and requests for materials should be addressed to T.I. (imura@nuis.ac.jp)

\section{Differences between chimpanzees and humans in visual temporal integration}

\author{
Tomoko Imura' \& Masaki Tomonaga² \\ ${ }^{1}$ Niigata University of International and Information Studies, 3-1-1, Mizukino, Nishi-ku, Niigata, 950-2292, Japan, 2Primate
Research Institute, Kyoto University 41-2, Kanrin, Inuyama, Aichi, 484-8506, Japan.
}

Humans have a superior ability to integrate spatially separate visual information into an entire image. In contrast, comparative cognitive studies have demonstrated that nonhuman primates and avian species are superior in processing relatively local features; however, animals in these studies were required to ignore local shape when they perceived the global configuration, and no studies have directly examined the ability to integrate temporally separate events. In this study, we compared the spatio-temporal visual integration of chimpanzees and humans by exploring dynamic shape perception under a slit-viewing condition. The findings suggest that humans exhibit greater temporal integration accuracy than do chimpanzees. The results show that the ability to integrate local visual information into a global whole is among the unique characteristics of humans. 
(a)

(b)
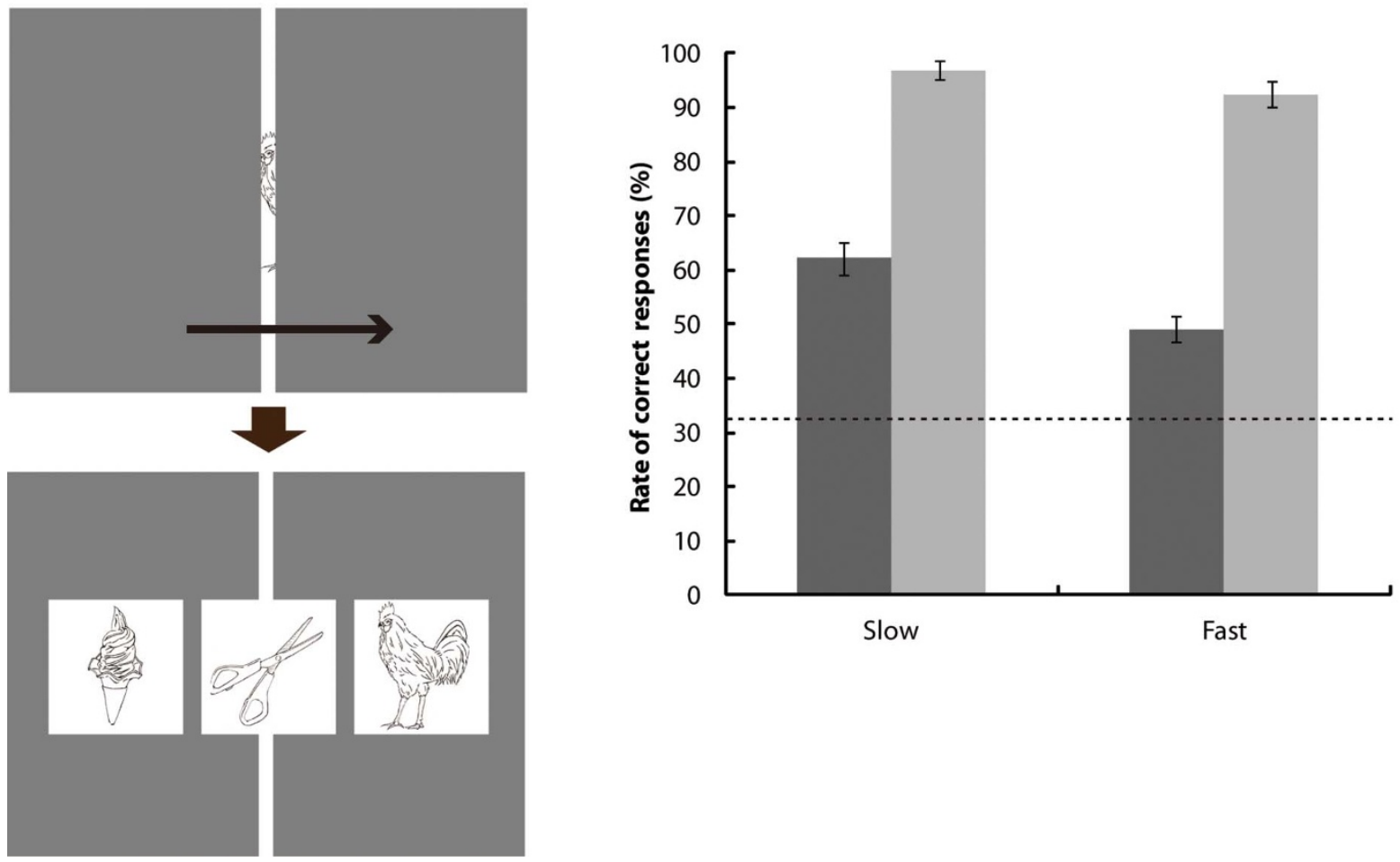

Figure $1 \mid$ (a) An example of the stimulus displays used in a slit-viewing task. A black line drawing is moving behind the slit from left to right, followed by three items presented on a monitor screen. (b) Performance of chimpanzees and humans under the slow and fast conditions.

(a)

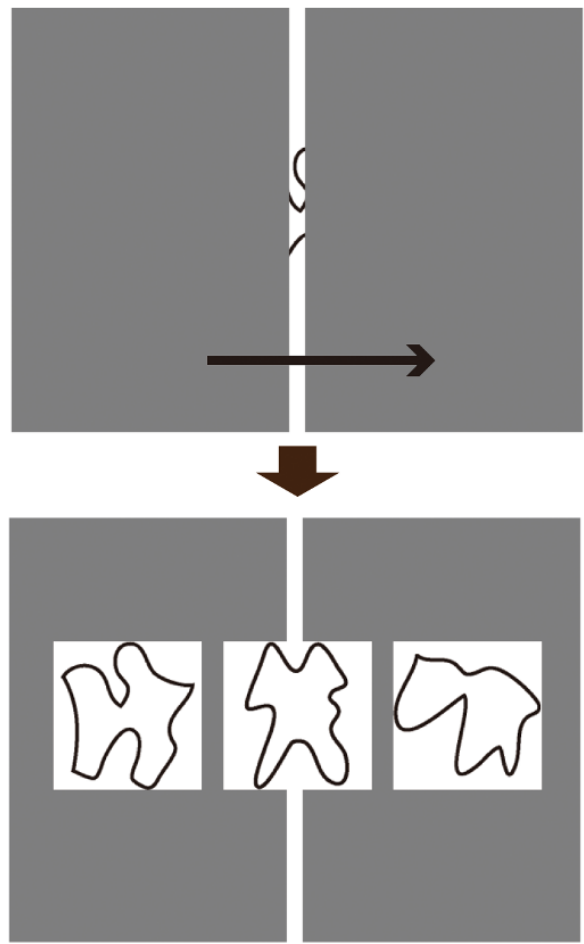

(b)

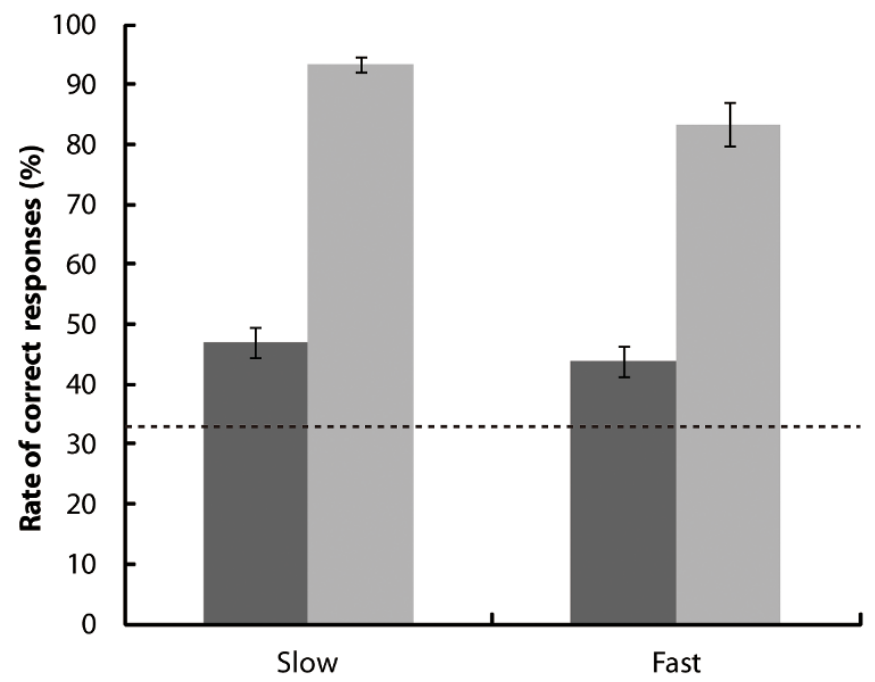

- Chimpanzees

- Humans
Chimpanzees

Humans

Figure $2 \mid$ (a) An example of the nonsense figures and stimulus displays used in a slit-viewing task. (b) Performance of chimpanzees and humans under the slow and fast conditions. 
Nonsense figures. The mean rates of correct responses were lower among chimpanzees than among humans (Fig. 2b). A two-way ANOVA with species $(2) \times$ speed $(2)$ revealed significant main effects of species $[F(1,10)=212.06, p<0.001]$ and speed $[F(1$, $10)=5.03, p<0.05]$. These findings suggest that the humans were better able to integrate local visual information into a whole global image than were chimpanzees. In a sequential presentation task, we examined whether the chimpanzees' inferior performance was due to preferential processing of local features.

\section{Discussion}

In this study, we compared the spatio-temporal visual integration of chimpanzees and humans by exploring dynamic shape perception under a slit-viewing condition. We found that both chimpanzees and humans integrated spatio-temporal visual information but that the extent of the integration differed between species. The findings with nonsense figures demonstrated that semantic processing couldn't account for the species differences. These findings are consistent with those of previous studies of spatial integration in nonhuman primates. Our results show that the ability to integrate motion and shape information may be more prominent in humans than chimpanzees.

Although the fundamental mechanism of motion perception in chimpanzees has not been studied well, some studies have found species differences in terms of motion integration processing ${ }^{27-29}$. For example, humans have a great advantage when detecting a stationary dot among consistently moving rather than randomly moving dots; however, chimpanzees have not shown any differences in performance based on motion coherence ${ }^{27}$. These findings suggest that humans are superior to chimpanzees with respect to organising coherently moving objects. As another example, humans observing the "stream-bounce display", which consists of two identical disks approaching each other, overlapping, and separating, tend to interpret this display as streaming rather than as bouncing, even though it can be interpreted in terms of both streaming and bouncing. In contrast, chimpanzees do not show a bias toward a "streaming" perception. This species difference can be explained in terms of abilities related to spatio-temporal integration ${ }^{28}$. The findings of dynamic shape perception under the slit-viewing condition extend the evidence for relative local biases in spatial and temporal processing by chimpanzees.

In humans, functional magnetic resonance imaging (fMRI) studies have shown that such spatio-temporal integration under a slitviewing condition evokes responses in the ventral occipital complex and motion comple ${ }^{26}$. These cortical networks are implicated in the other type of motion-defined grouping task in which the fragmented line drawings are integrated into one object ${ }^{30}$. Although the neurophysiological processes of nonhuman primates during slit viewing remain unclear, recent $\mathrm{fMRI}$ studies using the same paradigms and stimuli in humans and monkeys have shown pronounced differences in the middle part of the intra-parietal sulcus, which has been implicated in three-dimensional motion perception, including visuomotor control of actions ${ }^{31-33}$. Species differences between chimpanzees and humans reported in behavioural studies may carry further implications for neurophysiological research.

\section{Methods}

Participants. Eight humans (mean age, 21.4 years) participated in a slit-viewing task. All participants had normal or corrected-to-normal vision. Four chimpanzees ( $P a n$ troglodytes) (Ai, 33-year-old female; Ayumu, 9-year-old male; Cleo, 9-year-old female, and Pal, 9-year-old female) participated in a slit-viewing task. All chimpanzees lived in an enriched outdoor enclosure at the Primate Research Institute of Kyoto University with other group members. They were fed fruits, and vegetables three times daily during the experimental period. These chimpanzees have previously engaged in various kinds of computer-controlled perceptual and cognitive tasks ${ }^{27,29,34-36}$. The experimental protocol was approved by the Animal Welfare and Animal Care Committee of the Primate Research Institute of Kyoto University, and the chimpanzees were tested and cared for according to "The Guide for the Care and
Use of Laboratory Primates, 2nd edition" issued by the Ethics Committee of the Primate Research Institute of Kyoto University (2002). The human participants were undergraduate students, who participated in the experiment voluntarily. Informed consent was obtained from all human participants.

Apparatus. The experiments with chimpanzees and humans were conducted in an experimental booth $(1.8 \times 2.15 \times 1.75 \mathrm{~m})$ adjacent to the chimpanzee facility. Stimuli were presented on an 18.1-inch colour LCD monitor with a touch-screen device (Iiyama, A4146D) located $40 \mathrm{~cm}$ above the floor. The resolution of the monitor was $1,280 \times 1,024$ pixels with a refresh rate of $60 \mathrm{~Hz}$, and the viewing distance was approximately $40 \mathrm{~cm}$. The monitor was protected by a transparent Plexiglas panel, and participants could touch the monitor through an armhole (38.5 $\times 12 \mathrm{~cm}$ ). A food dispenser (Biomedica BUF-310) delivered rewards to the chimpanzees following each correct trial via food trays attached below the monitor. All experimental events and responses were controlled by a computer (HewlettPackard Compaq, PM215AV) located outside the experimental booth.

Materials. The stimuli were black-and-white line drawings or nonsense figures containing the images shown in Figures 1a and $2 \mathrm{a}$. The lines of figures were black $\left(0.04 \mathrm{~cd} / \mathrm{m}^{2}\right)$ and the background was white $\left(112 \mathrm{~cd} / \mathrm{m}^{2}\right)$. We used 40 types of objects and nonsense figures subtended approximately $6-7^{\circ}$ of visual angle ${ }^{25,26}$ (the original images were constructed by Snodgrass and Vanderwart ${ }^{37}$, and Endo et al. ${ }^{38}$ ). A grey surface $\left(74.68 \mathrm{~cd} / \mathrm{m}^{2}\right)$ with a slit in the centre was used to partially occlude the image. The widths of the slit were 6 pixels $\left(0.31^{\circ}\right), 18$ pixels $\left(0.93^{\circ}\right)$, and 30 pixels $\left(0.55^{\circ}\right)$. The image was translated at $14.3^{\circ} \mathrm{sec}$ under the fast condition and at $7.15^{\circ} / \mathrm{sec}$ under the slow condition. The item always moved from left to right once per trial.

Procedure. A line drawing of an object or nonsense figure moved behind a slit at either a slow or fast speed, followed by three items presented on a monitor screen. One of the three items was identical to a drawing that previously moved behind the slit. The task was to select this same drawing from among the three alternatives. Before testing, the chimpanzees were trained to choose the correct item under a condition in which whole images were presented in front of the slit. Each block consisted of 120 trials ( 40 items $\times 3$ slit widths) under each speed condition. It took the chimpanzees $2-7$ blocks to meet the criterion of a $>90 \%$ correct response rate during consecutive sessions.

Data from the trials with the narrowest slit were used for analyses. The remaining slit width conditions were provided for chimpanzees to keep the motivation for the task. However, it might be wide enough for humans to use a local cue. The trials with other conditions were excluded from the analysis. Data from 10 test blocks in total from all chimpanzees and one test block in total from all humans were used for analyses.

1. Matsuno, T. \& Fujita, K. A comparative psychophysical approach to visual perception in primates. Primates 50, 121-130 (2009).

2. Navon, D. Forest before trees: the precedence of global features in visual perception. Cognit. Psychol. 9, 353-383 (1977).

3. Cavoto, K. K. \& Cook, R. G. Cognitive precedence for local information in hierarchical stimulus processing by pigeons. J Exp Psychol Anim Behav Process. 27, 3-16 (2001).

4. Deruelle, C. \& Fagot, J. Visual search in global/local stimulus features in humans and baboons. Psychon. Bull. Rev. 3, 476-481 (1998).

5. Hopkins, W. D. \& Washburn, D. A. Matching visual stimuli on the basis of global and local features by chimpanzees (Pan troglodytes) and rhesus monkeys (Macaca mulatta). Anim. Cogn. 5, 27-31 (2002).

6. Spinozzi, G., De Lillo, C. \& Salvi, V. Local advantage in the visual processing of hierarchical stimuli following manipulations of stimulus size and element numerosity in monkeys (Cebus apella). Behav Brain Res. 166, 45-54 (2005).

7. Fagot, J. \& Tomonaga, M. Global and local processing in humans (Homo sapiens) and chimpanzees (Pan troglodytes): use of a visual search task with com- pound stimuli. J. Comp. Psychol. 113, 3-12 (1999).

8. Matsuno, T. \& Tomonaga, M. Global and local visual processing by chimpanzees (Pan troglodytes). Jpn J Psychon Sci. 25, 281-282 (2007).

9. Cook, R. G., Goto, K., \& Brooks, D. I. Avian detection and identification of perceptual organization in random noise. Behav Process. 69, 79-95 (2005).

10. Nakamura, N., Watanabe, S. \& Fujita, K. Pigeons perceive the EbbinghausTitchener circles as an assimilation illusion. J Exp Psychol Anim Behav Process. 34, 375-387 (2008)

11. Watanabe, S., Nakamura, N. \& Fujita, K. Pigeons perceive a reversed Zöllner illusion. Cognit 119, 137-141 (2011).

12. Fujita, K. \& Ushitani, T. Better living by not completing: a wonderful peculiarity of pigeon vision? Behav Processes 69, 59-66 (2005).

13. Ushitani, T. \& Fujita, K. Pigeons do not perceptually complete partly occluded photos of food: an ecological approach to the "pigeon problem" Behav Processes 69, 59-66 (2005).

14. Tanaka, H. \& Fujita, I. Global and local processing of visual patterns in macaque monkeys. Neuroreport 11, 2881-2884 (2000).

15. Neiworth, J. J., Gleichman, A. J., Olinick, A. S. \& Lamp, K. E. Global and local processing in adult humans (Homo sapiens), 5-year-oldchildren (Homo sapiens), and adult cotton-top tamarins (Saguinus oedipus). J Comp Psychol. 120, 323-330 (2006). 
16. Colombo, J., Mitchell, D., Coldren, J. T. \& Freeseman, L. J. Individual differences in infant visual attention: Are short lookers faster processors or feature processors? Child Dev 62, 1247-1257 (1991).

17. Frick, J. E., Colombo, J. \& Allen, J. R. Temporal sequence of global - local processing in 3-month-old infants. Infancy 1, 375-386 (2000).

18. Freeseman, L. J., Colombo, J. \& Coldren, J. T. Individual differences in infant visual attention: Four-month-olds' discrimination and generalization of global and local stimulus properties. Child Development 64, 1191-1203 (1993).

19. Guy, M. W., Reynolds, G. D. \& Zhang, D. Visual attention to global and local stimulus properties in 6-month-old infants: Individual differences and eventrelated potentials. Child development, (2013).

20. Mondloch, C. J., Geldart, S., Maurer, D. \& de Schonen, S. Developmental changes in the processing of hierarchical shapes continue into adolescence. J. Experimental Child Psychology 84, 20-40 (2003).

21. Helmholtz, H. von. Handbook of physiological optics: JS Southhall translation ( $3^{\text {rd }}$ ed.). New York: Dover (1867/1962).

22. Parks, T. E. Post-retinal visual storage. Am. J. Psychol. 78, 145-147 (1965).

23. Zollner, F. Uber eine neue Art anorthoscopischer Zerrbilder. Annalen der Physik und Chemie: Poggendorffs Annalen 117, 477-484 (1862).

24. Ağaoğlu, M. N., Herzog, M. H. \& Öğmen, H. Non-retinotopic feature processing in the absence of retinotopic spatial layout and the construction of perceptual space from motion. Vision Research 71, 10-17 (2012).

25. Nakano, T., Ota, H., Kato, N. \& Kitazawa, S. Deficit in visual temporal integration in autism spectrum disorders. Proceedings of the Royal Society B: Biological Sciences 277(1684), 1027-1030 (2010).

26. Yin, C., Shimojo, S., Moore, C. \& Engel, S. A. Dynamic shape integration in extrastriate cortex. Curr. Biol. 12, 1379-1385 (2002).

27. Matsuno, T. \& Tomonaga, M. Visual search for moving and stationary items in chimpanzees (Pan troglodytes) and humans (Homo sapiens). Behav Brain Res. 172, 219-232 (2006).

28. Matsuno, T. \& Tomonaga, M. Stream/bounce perception and the effect of depth cues in chimpanzees (Pan troglodytes). Atten Percept Psychophys. 73, 1532-1545 (2011).

29. Imura, T. \& Tomonaga, M. Moving shadows contribute to the corridor illusion in a chimpanzee (Pan troglodytes). Journal of Comparative Psychology 123, 280-286 (2009).

30. Ferber, S., Humphrey, G. K. \& Vilis, T. The lateral occipital complex subserves the perceptual persistence of motion-defined groupings. Cereb Cortex 7, 716-721 (2003)

31. Orban, G. A. et al. Similarities and differences in motion processing between the human and macaque brain: evidence from fMRI. Neuropsychologia 41, 1757-1768 (2003)

32. Orban, G. A. et al. Comparative mapping of higher visual areas in monkeys and humans. Trends Cogn. Sci. 8, 315-324 (2004).
33. Vanduffel, W. et al. Extracting 3D from motion: differences in human and monkey intraparietal cortex. Science 298, 413-415 (2002).

34. Imura, T., Tomonaga, M. \& Yagi, A. The effects of linear perspective on relative size discrimination in chimpanzees (Pan troglodytes) and humans (Homo sapiens). Behav Processes 77, 306-312 (2008).

35. Matsuzawa, T. The Ai project: historical and ecological contexts. Animal Cognition 6, 199-211 (2003).

36. Matsuzawa, T., Tomonaga, M. \& Tanaka, M. (Eds.). Cognitive development in chimpanzees. Tokyo: Springer-Verlag Tokyo (2006).

37. Snodgrass, J. G. \& Vanderwart, M. A standardized set of 260 pictures: norms for name agreement, image agreement, familiarity, and visual complexity. J. Exp. Psychol.: Hum. Learn. 6, 174-215 (1980).

38. Endo, N., Saiki, J., Nakao, Y. \& Saito, H. Perceptual judgments of novel contour shapes and hierarchical descriptions of geometrical properties. Jap. Psychol. Rev. 74, 346-353 (in Japanese) (2003).

\section{Acknowledgments}

This study was financially supported by the JSPS-MEXT Grants in Aid for Scientific Research $(196211,21830053,23135516$, and 23700312 to T.I., 20002001, and 23220006 to M.T.), Global COE Programs (A06, D07), and the Benesse Corporation. We thank T. Kaneko, I. Adachi, M. Hayashi, and T. Matsuzawa for their helpful support and suggestions We also thank the members of the Center for Human Evolution Modeling Research and the Language and Intelligence Section of the Primate Research Institute for technical advice and support and for their care of the chimpanzees. We appreciate T. Hasegawa for preparing figure 1

\section{Author contributions}

T.I. and M.T. were involved in study design; T.I. collected and analysed data; T.I. and M.T. discussed the results and wrote main manuscript text.

\section{Additional information}

Competing financial interests: The authors declare no competing financial interests. How to cite this article: Imura, T. \& Tomonaga, M. Differences between chimpanzees and humans in visual temporal integration. Sci. Rep. 3, 3256; DOI:10.1038/srep03256 (2013).

This work is licensed under a Creative Commons AttributionNonCommercial-NoDerivs 3.0 Unported license. To view a copy of this license, visit http://creativecommons.org/licenses/by-nc-nd/3.0 